\title{
The yeast Chs4 protein stimulates the trypsin- sensitive activity of chitin synthase 3 through an apparent protein-protein interaction
}

\author{
Naomi Ono, ${ }^{1}$ Tomio Yabe, ${ }^{1,2}$ Masayuki Sudoh, ${ }^{1}$ Tasuku Nakajima, ${ }^{2}$ \\ Toshiko Yamada-Okabe, ${ }^{3}$ Mikio Arisawa ${ }^{1}$ and Hisafumi Yamada-Okabe ${ }^{1}$
}

Author for correspondence: Hisafumi Yamada-Okabe. Tel: +8146747 2242. Fax: +81467465320. e-mail: hisafumi.okabe@roche.com

\footnotetext{
${ }^{1}$ Mycology Department, Nippon Roche Research Center, 200 Kajiwara, Kamakura, Kanagawa 247-8530, Japan

2 Division of Life Science, Graduate School of Agricultural Science, Tohoku University, Aoba-ku, Sendai 981-8555, Japan

3 Department of Hygiene, School of Medicine, Yokohama City University, 3-9 Fukuura, Kanazawaku, Yokohama 236-0004, Japan
}

\begin{abstract}
Inducible overexpression of the CHS4 gene under the control of the GAL1 promoter increased Chs3p (chitin synthase 3) activity in Saccharomyces cerevisiae several fold. Approximately half of the Chs3p activity in the membranes of cells overexpressing Chs4p was extracted using CHAPS and cholesteryl hemisuccinate. The detergent-extractable Chs3p activity appeared to be non-zymogenic because incubation with trypsin decreased enzyme activity in both the presence and absence of the substrate, UDP-Nacetylglucosamine. Western blotting confirmed that Chs3p was extracted from membranes by CHAPS and cholesteryl hemisuccinate and revealed that Chs4p was also solubilized using these detergents. Yeast two-hybrid analysis with truncated Chs4p demonstrated that the region of Chs4p between amino acids 269 and 563 is indispensable not only for eliciting the non-zymogenic activity of Chs3p but also for binding of Chs4p to Chs3p. Neither the EF-hand motif nor a possible prenylation site in Chs4p was required for these activities. Thus, it was demonstrated that stimulation of non-zymogenic Chs3p activity by Chs4p requires the amino acid region from 269 to 563 of Chs4p, and it seems that Chs4p activates Chs3p through protein-protein interaction.
\end{abstract}

Keywords: yeast, chitin synthase 3, overexpression, protein-protein interaction

\section{INTRODUCTION}

In Saccharomyces cerevisiae, chitin deposition at the bud neck is important for the normal budding process, and inhibition of chitin synthesis causes a defect in septum formation. Three yeast chitin synthases, Chs1p, Chs $2 p$ and Chs3p, have been characterized by generating mutants affected in these proteins. Chs1p repairs damaged chitin, counter-balancing the activities of chitinase (Bulawa et al., 1986; Cabib et al., 1989, 1992), and Chs $2 p$ is responsible for formation of the primary septum (Sburlati \& Cabib, 1986; Shaw et al., 1991; Silverman, 1989; Silverman et al., 1988). Chs3p synthesizes most of the cell-wall chitin and also links chitin to the non-reducing end of $1,3-\beta$-glucan by a $\beta-1,4-$ linkage (Kollar et al., 1995; Valdivieso et al., 1991). Disruption of CHS1 did not result in any significant morphological alterations, but simultaneous disruption of CHS2 and CHS3 is lethal (Shaw et al., 1991), indicating the importance of chitin for viability in yeast. The level of Chs $2 p$ is regulated during the cell cycle. Chs $2 p$ is localized in the bud neck at the end of mitosis, and then degraded in a Pep4p-dependent manner (Chuang \& Schekman, 1996). Chs3p is a metabolically stable protein that appears at a ring on the cell surface at bud emergence and then accumulates in the mother-bud neck of small-budded cells (DeMarini et al., 1997; Santos \& Snyder, 1997; Ziman et al., 1996). Chs3p function requires several cellular factors, including the products of the genes CHS4, CHS5, CHS6, MYO2 and TIG1 (Bulawa, 1992; DeMarini et al., 1997; Holthuis et al., 1998; Santos \& Snyder, 1997; Santos et al., 1997; Trilla et al., 1997; Ziman et al., 1998). CHS4 encodes a protein that directs the correct localization of Chs3p at the mother-bud neck by binding Bni4p as well as one of the septins, Cdc10p (DeMarini et al., 1997). Chs5p, Myo2p and Tig1p play important roles in the polarized distribution of Chs3p (Holthuis et al., 1998; Santos \& Snyder, 1997), and Chs6p mediates the translocation of Chs3p from the chitosome to the cell surface (Ziman et al., 1998). Among these genes, mutation in either CHS4 or CHS5 decreases Chs3p activity and cell-wall chitin content (Bulawa, 1992; Santos et al., 1997; Trilla et al., 1997). 
Previously, we used mutation analyses to demonstrate the residues important for Chs 2 activity. Chs $2 p$ contains two regions called con 1 and con2, which are highly conserved among various chitin synthases, in the middle and at the C-terminus of the protein. Region con1 contains two amino acid sequence motifs, Glu-Asp-Arg and Gln-Arg-Arg-Arg-Trp, and con2 has one motif, His-Asp-X-X-Trp-X-Thr, where $\mathrm{X}$ represents variations. Because enzyme activity was severely impaired by mutations of the conserved amino acids in these motifs, they are presumed to contribute to the active site of the enzyme (Nagahashi et al., 1995; Yabe et al., 1998). In fact, mutations of some of these conserved amino acids in Chs3p also diminished the activity and functionality of the enzyme (Cos et al., 1998).

In contrast to the sequence conservation in the probable active sites among the three yeast chitin synthases, Chs $3 p$ activity is regulated by a mechanism different from those for Chs1p and Chs2p. Firstly, overexpression in yeast of CHS1 or CHS2 led to an increase in chitin synthase activity, whereas overexpression of CHS3 alone did not (Bulawa et al., 1986; Nagahashi et al., 1995; Valdivieso et al., 1991). Introduction into yeast of additional copies of CHS4, however, enhances Chs3p activity and increases the amount of cell-wall chitin (Bulawa 1993; Trilla et al., 1997), suggesting that Chs3p activity requires CHS4 as a positive regulator. Secondly, two types of Chs3p activities, trypsin-dependent (zymogenic) and trypsin-sensitive (non-zymogenic), are present in membranes (Choi et al., 1994), although both Chs1p and Chs2p are known only as zymogens (Cabib et al., 1996). The zymogenic activity of Chs3p is not detergent-extractable, and Chs3p needs to be in complex with the substrate during activation by trypsin (Choi et al., 1994). Zymogenic Chs3p activity was detected only after the membranes were treated with detergents, and a certain level of detergent-extractable non-zymogenic Chs $3 p$ activity might be expected to be present in the membranes (Choi et al., 1994). Such non-zymogenic Chs3p activity, however, has never been recovered and confirmed.

In this paper we show that the non-zymogenic activity of Chs $3 p$ can be extracted with CHAPS and cholesteryl hemisuccinate from membranes prepared from cells overexpressing CHS4, and that the association of Chs3p and Chs $4 p$ is important for non-zymogenic Chs $3 p$ activity. In addition, the region of Chs $4 p$ required for the activity of, and binding to, Chs $3 p$ is demonstrated by truncation of CHS4 and yeast two-hybrid analysis.

\section{METHODS}

Plasmids and yeast strains. The entire ORFs of $S$. cerevisiae CHS3 and CHS4 were amplified by PCR and cloned at the $\mathrm{XbaI}$ site (CHS3) and between the BamHI and XbaI sites (CHS4) located downstream of the GAL1 promoter of pYES2, which carries $U R A 3$ as a selection marker (Invitrogen), creating pYES-CHS3 and pYES-CHS4, respectively. To detect Chs4p, the triple c-Myc-sequence tag was inserted just after the first ATG codon within the CHS4 ORF. The primers used for PCR were 5'-GATCGCGGATCCGCGATGGAACAAAAGCTTATTTCTGAAGAAGACTTGGCAAGTTCACCGCAGGTACATCCA-3' and 5'-ATCTAGTGCTCTAGATTACATAATTACACAGTC-3' for CHS4, and 5'-GAACACGCAGCTAGCATGGAACAAAAGCTTATTTCTGAAGAAGACTTGAATCACCTGGATGTTTTACC-3' and 5'GACCGTTCAGCTAGCCTATGCAACGAAGGAGTCACTTTC-3' for CHS3. These plasmids were transfected into the haploid S. cerevisiae strain YMS120 (MATa ade2 trp1 leu2 ura3 his3 chs14::ADE2 chs24::HIS3), in which the $2 \cdot 1 \mathrm{~kb}$ NcoI-NcoI region of the chromosomal CHS1 and the $1.1 \mathrm{~kb} B g l \mathrm{II}-\mathrm{Bg} l \mathrm{II}$ region of the chromosomal CHS2 were replaced by $A D E 2$ and $H I S 3$, respectively, by the lithium acetate method (Ito et al., 1983). For the simultaneous overexpression of CHS3 and CHS4, YpL-CHS3, where the CHS3 ORF was cloned at the XbaI site (downstream of the GAL1 promoter) of YpLX harbouring LEU2 (Nagahashi et al., 1995), was also created, and was transfected into YMS120 along with pYES-CHS4. The $\mathrm{Ura}^{+}$(for the pYES2based plasmids), $\mathrm{Leu}^{+}$(for the YpLX-based plasmids) and $\mathrm{Ura}^{+} \mathrm{Leu}^{+}$(for double transfection) transformants were cultured at $30^{\circ} \mathrm{C}$ in yeast nitrogen base (Difco) supplemented with glucose and necessary amino acids, and the expression of Chs3p and Chs $4 p$ was induced in the same medium, but containing galactose instead of glucose, at $30^{\circ} \mathrm{C}$ for $12 \mathrm{~h}$. A series of truncated CHS4 genes, which would express $\mathrm{N}$ terminally and/or C-terminally truncated Chs4 proteins, was also created by PCR and cloned between the Bam HI and XbaI sites of pYES2.

To examine the functionality of CHS4, the haploid $S$. cerevisiae strain YMS1 (MATa ade2 trp1 leu2 ura3 his3 chs $44:: A D E 2)$, in which the $4 \cdot 2 \mathrm{~kb}$ AflII-NheI region of the chromosomal CHS4 was replaced by ADE2, was transformed by pYES-CHS4 carrying the wild-type or a truncated CHS4 and selected by uracil auxotrophy in the presence of galactose. The resulting $\mathrm{Ura}^{+}$transformants were collected and spread on plates containing galactose and $2 \mathrm{mg}$ Calcofluor white $\mathrm{ml}^{-1}$.

Membrane preparation and chitin synthase assay. Total membranes were prepared from yeast cells as described previously (Nagahashi et al., 1995). To extract Chs3p, $20 \mathrm{mg}$ protein from total membranes was suspended in $2 \mathrm{ml}$ of a buffer containing $20 \mathrm{mM}$ Tris/ $\mathrm{HCl}(\mathrm{pH} 7 \cdot 6), 160 \mathrm{mM} \mathrm{NaCl}$, $20 \mu \mathrm{M}$ GTP- $\gamma \mathrm{S}, 5 \mathrm{mM}$ dithiothreitol, $160 \mathrm{mM} \mathrm{NaCl}$, $33 \%(\mathrm{v} / \mathrm{v})$ glycerol, $0.5 \%$ (v/v) CHAPS, and $0.1 \%(\mathrm{w} / \mathrm{v})$ cholesteryl hemisuccinate, and left on ice for $30 \mathrm{~min}$. After centrifugation at $200000 \mathrm{~g}$ for $30 \mathrm{~min}$, the supernatants were collected and used as the membrane extracts (S100). The precipitates were also suspended in an equal volume of the same buffer and used as the extracted membranes (P100). In some experiments, the total membranes and membrane extracts were incubated with the indicated concentrations of trypsin in the presence or absence of $1 \mathrm{mM}$ UDP-N-acetylglucosamine (UDP-GlcNAc). After incubation at $30^{\circ} \mathrm{C}$ for $15 \mathrm{~min}$, trypsin was inactivated by the addition of soybean trypsin inhibitor at a concentration twice that of the trypsin added to the reaction mixture.

Unless otherwise specified, chitin synthase assays were performed in a standard $100 \mu \mathrm{l}$ reaction mixture containing $30 \mathrm{mM}$ Tris/ $\mathrm{HCl}, \mathrm{pH} 7 \cdot 5,5 \mathrm{mM} \mathrm{MgCl}_{2}, 32 \mathrm{mM}$ GlcNAc, $1.0 \mathrm{mM}\left[{ }^{3} \mathrm{H}\right]$ UDP-GlcNAc (specific activity 10000 d.p.m. $\mathrm{nmol}^{-1}$ ), the indicated amount of total membranes or membrane extract at $30^{\circ} \mathrm{C}$ for $60 \mathrm{~min}$, and acid-insoluble radioactivity was measured using a scintillation counter. 
(a)

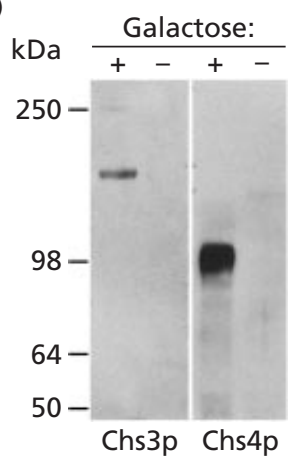

(b)

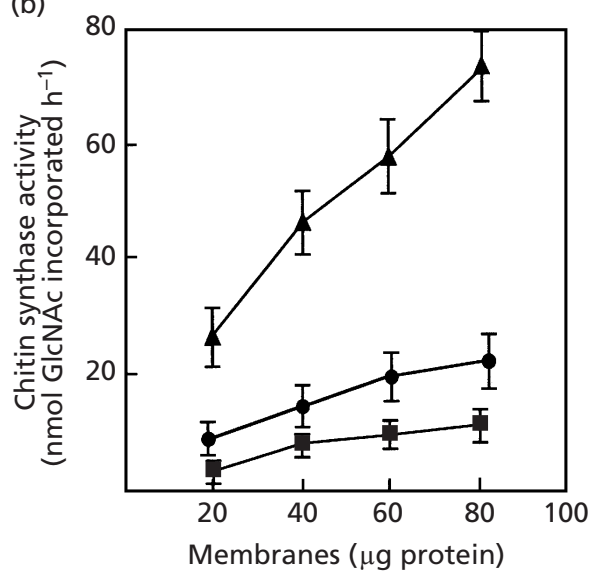

(c)

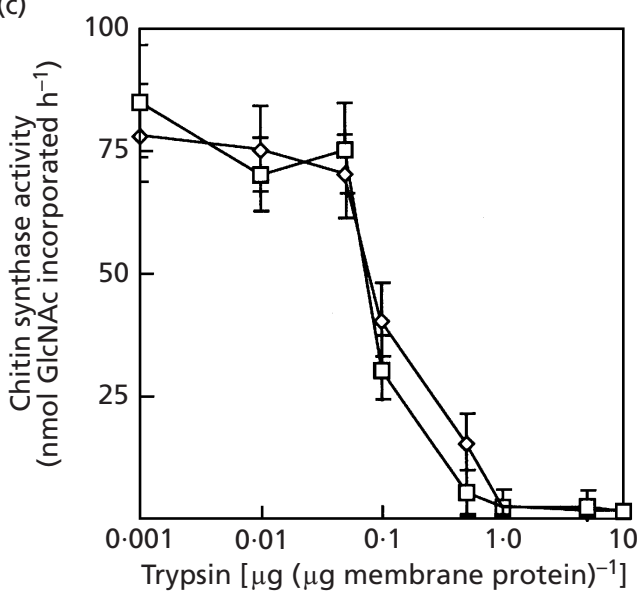

Fig. 1. Increase in Chs3p activity following overexpression of Chs4p. (a) Yeast cells, carrying pYES-CHS3 or pYES-CHS4, were grown to mid-exponential phase, then further cultured for $12 \mathrm{~h}$ in medium containing glucose $(-)$ or galactose (+). Approximately $100 \mu \mathrm{g}$ membrane protein was fractionated by SDS-PAGE and transferred to a PVDF membrane. For cells harbouring pYES-CHS3, blots were incubated with anti-Chs3p antibody (Chs3p); for cells harbouring pYES-CHS4 they were incubated with anti-c-Myc antibody (Chs4p). (b) Chs3p activity in cells overexpressing Chs3p or Chs4p. Yeast cells in mid-exponential phase were further cultured in medium containing galactose for $12 \mathrm{~h}$ before harvest. Chs3p activity was determined in membranes from YMS120 cells that had been transformed with pYES2 (ם), pYES-CHS3 (0) or pYES-CHS4 (A). (c) Effect of trypsin on Chs3p activity. Chs3p activity was determined with approximately $80 \mu \mathrm{g}$ protein from membranes of YMS120 cells that had been transformed with pYES-CHS4. The yeast cells were cultured as described in (b). Before the assay, the membranes were treated with the indicated concentrations of trypsin in the absence $(\diamond)$ or the presence $(\square)$ of the substrate. The error bars represent mean \pm SD for three independent experiments.

Western blotting. The indicated amounts of total membranes or membrane extract were suspended in a loading buffer for SDS-PAGE (Laemmli, 1970) and heated at $90^{\circ} \mathrm{C}$ for $2 \mathrm{~min}$. The proteins were then fractionated by SDS-PAGE (Laemmli, 1970), transferred electrophoretically to a PVDF membrane (Sambrook et al., 1989), and reacted with an anti-c-Myc monoclonal antibody (clone 9E10, Oncogene Science) or an anti-Chs3p polyclonal antibody, and then with horseradishperoxidase conjugated protein A (Amersham). The proteins which hybridized with the anti-c-Myc antibody or the antiChs3p antibody were detected by using an ECL-protein detection kit (Amersham). To generate the anti-Chs3p antibody, the Chs3p fragment that encompasses amino acids 255-434 was expressed in Escherichia coli as a fusion with glutathione $S$-transferase (GST). Rabbits were immunized with the purified protein, and the IgG fractions that contained the anti-Chs $3 p$ antibody were purified by affinity column chromatography using protein A Sepharose CL-4B (Pharmacia). To remove anti-GST antibodies from the IgG fractions, the purified $\operatorname{IgG}$ fractions were repeatedly loaded onto GST columns in which GST was bound to glutathione Sepharose beads, and the flow-through fractions, which contained no detectable anti-GST antibody, were pooled and used as the anti-Chs3p antibody preparation.

Yeast two-hybrid analysis. The entire ORF of CHS3 was cloned between the EcoRI and BamHI sites of pGAD424, generating a plasmid that would express Chs3p as a fusion protein with the transactivation domain of Gal4p. Regions of the CHS4 ORF encoding truncated Chs4 proteins were also cloned between the Bam HI and SalI sites of pGBT9 (Clontech) to express these products as fusion proteins with the DNAbinding domain of Gal4p. The resulting plasmids were then transformed into $S$. cerevisiae strain HF7c (MATa ura3-52 his3-200 lys2-801 ade2-101 trp1-901 leu2-3,112 gal4-542 gal80-
538 LYS2:: GAL1-HIS3 URA3::(GAL4 17-MERS) 3 -CYC1$L A C Z$ ), where HIS3 gene expression was dependent on both the DNA-binding and transactivation domains of Gal4p (Feilotter et al., 1994). After the transformation of HF7c, the $\mathrm{Leu}^{+} \mathrm{Trp}^{+}$transformants were collected and tested for their ability to grow in the absence of histidine.

\section{RESULTS}

\section{Involvement of CHS4 in Chs3p activity}

It has been shown previously that introduction into yeast of additional copies of CHS4 increases Chs3p activity and the total amount of chitin synthesized (Trilla et al., 1997). We sought to establish whether the inducible overexpression of CHS4 by the GAL1 promoter would enhance Chs3p activity. We used YMS120 cells, which lack functional CHS1 and CHS2 alleles, as the host, to eliminate Chs1p and Chs $2 \mathrm{p}$ activities during the assay for Chs $3 p$ activity. To confirm the induced expression of Chs3p and Chs4p, an anti-Chs3p polyclonal antibody was generated, and Chs4p was $\mathrm{N}$ terminally fused with the c-Myc-sequence tag. There were no detectable amounts of Chs3p and Chs4p when YMS120 cells bearing either pYES-CHS3 or pYESCHS4 were cultured in medium containing glucose (Fig. 1a). However, cultivation of these cells in galactose medium strongly increased the levels of the Chs3 and Chs4 proteins (Fig. 1a). The sizes of Chs3p and Chs $4 p$ detected by Western blotting were larger than would have been expected from their amino acid sequences (132 kDa for Chs3p, $77 \mathrm{kDa}$ for Chs4p) (Fig. 1a). These larger sizes are presumably due to glycosylation of Chs $3 p$ and Chs4p, because endoglycosidase-H treatment 
(a)

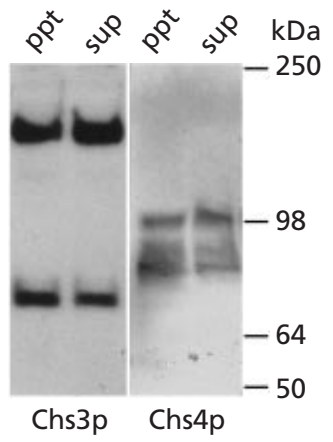

(b)

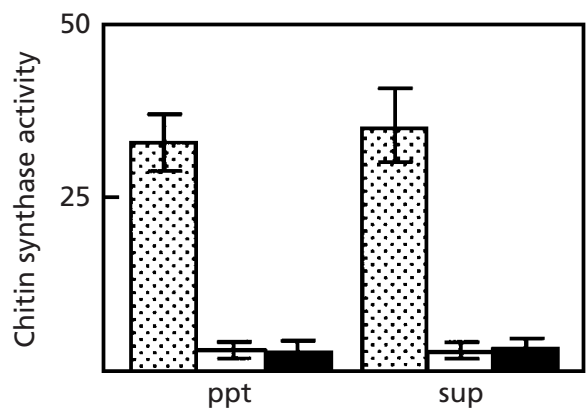

Fig. 2. Extraction of active Chs $3 p$ from membranes. Yeast cells that had been transformed with YpL-CHS3 and pYES-CHS4 were grown to mid-exponential phase then further cultured for $12 \mathrm{~h}$ in medium containing galactose to induce the expression of Chs3p and Chs4p. (a) Membranes were prepared, incubated in a buffer containing CHAPS and cholesteryl hemisuccinate and centrifuged as described in Methods. Twenty-five microlitres of the S100 (sup) and P100 (ppt) fractions of the membrane extracts were fractionated by SDS-PAGE, transferred to a PVDF membrane and hybridized with the anti-Chs3p antibody (Chs3p) or the anti-c-Myc antibody (Chs4p). (b) Effect of trypsin on Chs3p activity. Chs3p activity in P100 (ppt) and S100 (sup) fractions of membrane extracts was determined for $80 \mu \mathrm{l}$ of each fraction; the values plotted are nmol GlcNAc incorporated $\mathrm{h}^{-1}$. Before the assay, S100 and P100 fractions were untreated (dotted bars) or treated with trypsin in the absence (white bars) or the presence (black bars) of the substrate. For trypsin treatment, $1.0 \mu \mathrm{g}$ trypsin was added per $\mu \mathrm{g}$ protein of each fraction. The error bars represent mean \pm SD for three independent experiments.

gave rise to faster-migrating bands (data not shown). Consistent with a previous report (Trilla et al., 1997), overexpression of Chs $4 p$ alone led to a several-fold increase in Chs $3 p$ activity in the total membranes, whereas overexpression of Chs3p led to a much smaller increase in Chs3p activity (Fig. 1b). This confirms that there is a limiting factor for Chs $3 p$ expression and that Chs $4 p$ positively regulates enzyme activity.

There are two types of Chs3p activities, zymogenic and non-zymogenic (Choi et al., 1994). Therefore, we examined whether the increased Chs3p activity caused by the overexpression of Chs $4 p$ is further increased by trypsin treatment. When the total membranes prepared from the cells overexpressing Chs $4 p$ were treated with trypsin, the Chs3p activity declined as trypsin concentration increased. Moreover, addition of UDPGlcNAc during the trypsin treatment did not protect the enzyme from trypsin (Fig. 1c). This suggests that, most, if not all, of the Chs4p-elicited Chs3p activity is nonzymogenic. According to Choi et al. (1994), nonzymogenic Chs3p activity can be released from membranes with detergents, such as CHAPS, though such detergent-extractable Chs3p activity has never been confirmed by others. We also looked for detergents that could extract Chs $3 p$ activity from membranes and found that about half of the Chs3p activity in membranes from cells that contained an increased level of Chs $4 p$ was extracted from the membranes by the combination of CHAPS and cholesteryl hemisuccinate (Fig. 2). Although some degraded protein bands appeared after the membranes were treated with the detergents (Fig. 2a), the detergent-extracted Chs3p retained enzyme activity (Fig. $2 \mathrm{~b})$. Consistent with the non-zymogenic property of Chs $4 p$-induced Chs $3 p$ activity in membranes, the detergent-extracted Chs3p activity was trypsin-sensitive even in the presence of UDP-GlcNAc (Fig. 2b). Together, these results demonstrate that the Chs4p-elicited Chs $3 p$ activity is non-zymogenic.

\section{Region of Chs4p essential for Chs3p activity}

The pathogenic fungus Candida albicans also harbours a CHS4 homologue (GenBank accession number AB003310). The amino acid sequence of $S$. cerevisiae Chs $4 p$ is highly homologous to that of $C$. albicans Chs4p in the C-terminal two-thirds, but the $\mathrm{N}$-terminal one-third is rather divergent (Sudoh et al., 1999). This prompted us to determine the region of Chs $4 p$ that is important for Chs3p activity. A series of truncated CHS4 genes was created and expressed in YMS120 cells (Fig. 3). As shown in Fig. 4, deletion of either the Nterminal 268 amino acids or the C-terminal 134 amino acids only partially diminished the ability of Chs4p to elicit the Chs $3 p$ activity in the total membranes of YMS120 cells. Neither the EF-hand motif nor the potential prenylation site of Chs $4 p$ was essential to stimulate Chs3p activity, because Chs4(269-563)p still significantly enhanced Chs3p activity (Fig. 4a). Further deletion from the C-terminus, however, nearly completely abolished the ability of Chs4p to elicit Chs3p activity; Chs4(269-445)p was no longer capable of increasing the Chs3p activity in YMS120 cells (Fig. 4a).

We also examined the effects of the $\mathrm{N}$-terminal and Cterminal truncations of CHS4 on the sensitivity of yeast cells to Calcofluor white. Cells of YMS1, which carries no functional CHS4 allele, were resistant to Calcofluor white, because of a decreased level of cell-wall chitin (Sudoh et al., 1999). Introduction of the wild-type CHS4 into the YMS1 cells restored the sensitivity to Calcofluor white (Fig. 4b). Deletion of the C-terminal 252 amino acids of Chs $4 p$ completely abolished the ability of the protein to confer Calcofluor-white-sensitive growth on YMS1 cells, while deletion of the N-terminal 268 amino acids and C-terminal 134 amino acids did not (Fig. 4b). From these results, we concluded that the region of Chs $4 p$ that is essential for the elicitation of Chs3p activity resides in the amino acid positions between 269 and 563. 


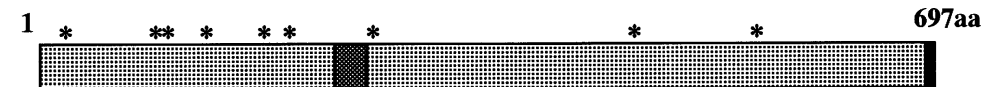

EF-hand motif

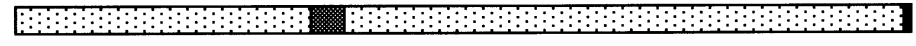

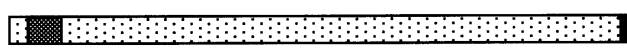

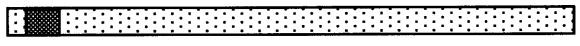

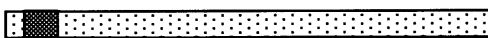

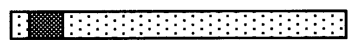

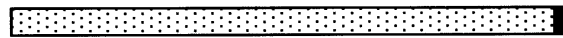

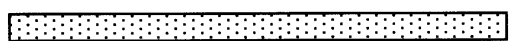

A:

:
Mutant CHS4

CHS4(1-697)/WT

CHS4(214-697)

CHS4(214-610)

CHS4(214-563)

CHS4(214-445)

CHS4(269-697)

CHS4(269-610)

CHS4(269-563)

CHS4(269-445)

* : potential $\mathrm{N}$-glycosylation site

CVIM: potential prenylation site

Fig. 3. Truncated CHS4 genes encoding the $\mathrm{N}$-terminally and C-terminally truncated Chs4 proteins used in this study. Each mutant was created by PCR and expressed under the control of the GAL1 promoter in pYES2. The mutant genes were designated according to the corresponding amino acid positions in the wild-type Chs4p. CHS4(1-697)/WT represents the wild-type CHS4, and the predicted domain structure of the wild-type Chs4p is shown at the top.

\section{Region of Chs4p that is essential for binding to Chs3p}

It has been shown that Chs4p is physically associated with Chs3p and Bni4p and that this interaction is necessary for the recruitment of Chs $3 p$ to the plasma membrane (DeMarini et al., 1997). Therefore, we wondered whether the association of Chs3p and Chs4p is also important for the stimulation of Chs3p activity. The ability of Chs $4 p$ to interact with Chs3p was confirmed by yeast two-hybrid analysis using the truncated CHS4 genes listed in Fig. 3. As shown in Fig. 5, the ability of the Chs $4 p$ fragments to interact with Chs3p correlated well with their capacity to stimulate Chs3p activity in the YMS120 cells and to confer Calcofluor sensitivity on the YMS1 cells (Fig. 4). Thus, Chs4(214445)p and Chs4(269-445)p, which failed to enhance Chs3p activity and restore Calcofluor-white-sensitive growth, were unable to directly interact with Chs3p (Fig. 5). In contrast, all of the other fragments of Chs4p which elicited these responses interacted directly with Chs3p. These results strongly support the idea that Chs4p stimulates the non-zymogenic activity of Chs3p by binding with it.

\section{DISCUSSION}

In this paper, we demonstrate that Chs $4 p$ stimulates the non-zymogenic activity of Chs3p which can be extracted from membranes using CHAPS and cholesteryl hemisuccinate. In addition, we have shown that the region of Chs $4 p$ which is necessary for the stimulation of nonzymogenic Chs $3 p$ activity resides between amino acids 269 and 563. Only this region was required for binding of Chs3p to Chs4p, and taken together these data suggest that the formation of a complex between Chs3p and Chs $4 p$ leads to activation of Chs $3 p$. About half of the Chs3p activity remained in membranes after the detergent treatment and could not be easily extracted even by repeated incubation of membranes with detergent. Thus, two different types of non-zymogenic Chs3p, CHAPS-extractable and non-CHAPS-extractable, may exist. As mentioned before, Choi et al. (1994) demonstrated that a certain level of Chs3p activity in membranes is attributable to the zymogenic activity of Chs3p, and that such zymogenic activity is not extracted by detergents. The ratio and physiological significance of zymogenic and non-zymogenic Chs3p activities, however, remain elusive.

Chs $4 p$ contains the amino acid sequence motifs characteristic of EF-hand calcium-binding sites and prenylation sites in the middle and at the C-terminus, respectively (Kawamoto et al., 1992). Experiments involving the truncation of Chs $4 p$ revealed that neither the EF-hand motif nor the prenylation site of Chs $4 p$ was essential for Chs3p-binding and stimulation of non-zymogenic Chs $3 \mathrm{p}$ activity. The real roles of these sequence motifs in Chs4p function remain to be elucidated; they may be required for other roles of Chs $4 \mathrm{p}$ such as resistance to Kluyveromyces lactis toxin (Kawamoto et al., 1992; Takita \& Castilho-Valvicius, 1993).

DeMarini et al. (1997) also demonstrated the physical association of Chs3p and Chs4p by yeast two-hybrid analysis. According to their results, the C-terminal 86 amino acids of Chs $4 p$ play an important role in binding to Chs3p because Chs4p lacking the C-terminal 86 amino acids interacted only weakly with Chs3p (DeMarini et al., 1997). It was also demonstrated that the Cterminal region of Chs $4 \mathrm{p}$ was crucial for the association 
(a)

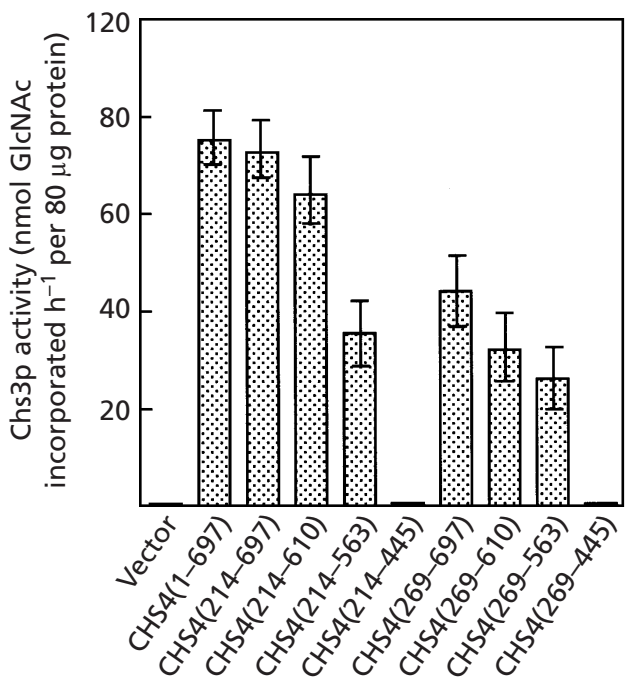

(b)

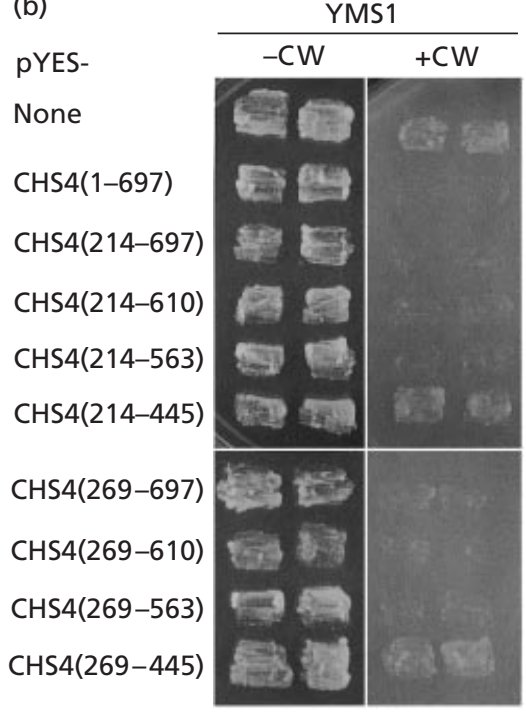

Fig. 4. Chs $3 p$ activity of cells overexpressing the truncated Chs4 proteins. (a) YMS120 cells that had been transformed with pYES2 carrying the indicated CHS4 genes were grown to midexponential phase then further cultured for $12 \mathrm{~h}$ in medium containing galactose to induce the expression of the truncated Chs4 proteins. Chs $3 p$ activity was determined using approximately $80 \mu \mathrm{g}$ membrane protein. The error bars represent mean $\pm S D$ for three independent experiments. (b) YMS1 cells that had been transformed with pYES2 carrying the indicated CHS4 genes were spread on agar plates containing galactose. Two independent colonies from each transformation were further cultured for $3 \mathrm{~d}$ in the absence $(-\mathrm{CW})$ or presence $(+\mathrm{CW})$ of $2 \mathrm{mg}$ Calcofluor white $\mathrm{ml}^{-1}$.

with Bni4p, which directs the correct localization of Chs3p at the bud neck through interaction with Cdc10p (DeMarini et al., 1997). In this study, we have shown that the C-terminal 134 amino acids of Chs4p are not essential for Chs $3 p$ activity, cellular chitin synthesis or binding to Chs3p. Because we judged association of the Chs $4 p$ fragments with Chs $3 p$ based on the histidine

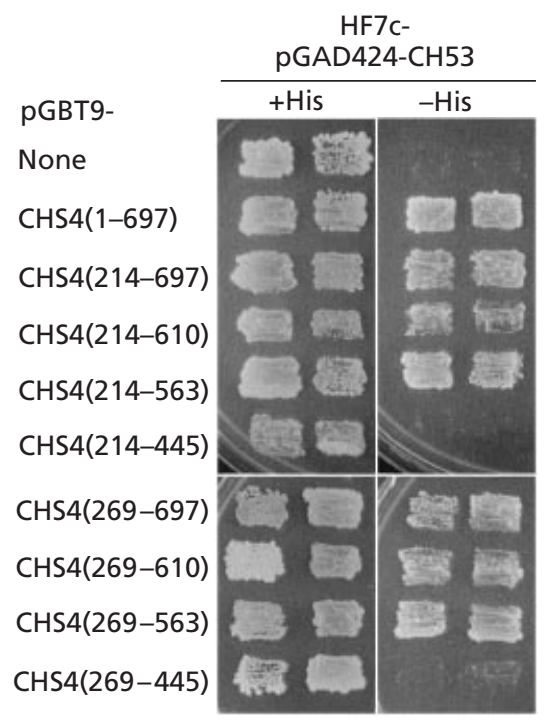

Fig. 5. Yeast two-hybrid analysis of the interaction of Chs3p with Chs4p. Yeast HF7c cells that were transformed with pGBT9 bearing the indicated CHS4 genes were further transfected with pGAD424 carrying CHS3. Two independent colonies from each transformation were collected and further cultured on agar plates with (+ His) or without (-His) histidine for $3 \mathrm{~d}$. The capacity of transformed strains to grow in the absence of histidine was dependent upon the interaction of Chs3p with Chs4p.

auxotrophy of transformants, it was difficult to determine the binding affinities of the truncated Chs4 proteins with Chs $3 p$ quantitatively. Nevertheless, our results and those of DeMarini et al. (1997) imply that a weak association of Chs4p with Chs3p is sufficient to elicit Chs3p activity.

The mechanism underlying the stimulation of Chs $3 p$ activity by Chs $4 p$ remains unclear. One possibility is that Chs $4 p$ stabilizes and/or increases the amount of Chs3p, although this is rather unlikely for the following reasons. Firstly, Chs3p is a stable protein with a half-life in excess of $10 \mathrm{~h}$ in vivo (Chuang \& Schekman, 1996). Secondly, overexpression of CHS3 alone increased the amount of Chs3p in membranes, yet Chs3p activity remained very low. Another possibility is that Chs $4 p$ affects the conformation of Chs $3 p$ and converts an inactive form of Chs3p into an active form. This seems more likely, because the simultaneous overexpression of CHS3 and CHS4 did not significantly increase the chitin synthase activity detected as compared to that of cells overexpressing CHS4 alone (data not shown). Thus, the expression of non-zymogenic Chs3p activity would appear to depend directly on the amount of Chs $4 p$ present.

\section{ACKNOWLEDGEMENTS}

We thank S. B. Miwa for reading the manuscript. This work was supported in part by Human Science funds to Nippon Roche Research Center and a grant from the Ministry of Education, Science and Culture of Japan to T.N. N. Ono and T. Yabe contributed equally to this work. 


\section{REFERENCES}

Bulawa, C. E. (1992). CSD2, CSD3, and CSD4, genes required for chitin synthesis in Saccharomyces cerevisiae: the CSD2 gene product is related to chitin synthases and to developmentally regulated proteins in Rhizobium species and Xenopus laevis. Mol Cell Biol 12, 1764-1776.

Bulawa, C. E. (1993). Genetics and molecular biology of chitin synthesis in fungi. Annu Rev Microbiol 47, 505-534.

Bulawa, C. E., Slater, M., Cabib, E., Au-Young, J., Sburlati, A., Adair, W. L., Jr \& Robbins, P. W. (1986). The S. cerevisiae structural gene for chitin synthase is not required for chitin synthesis in vivo. Cell 46, 213-225.

Cabib, E., Sburlati, A., Bowers, B. \& Silverman, S. J. (1989). Chitin synthase 1, an auxiliary enzyme for chitin synthesis in Saccharomyces cerevisiae. J Cell Biol 108, 1655-1672.

Cabib, E., Silverman, S. J. \& Shaw, J. A. (1992). Chitinase and chitin synthase 1: counterbalancing activities in cell separation of Saccharomyces cerevisiae. J Gen Microbiol 138, 97-102.

Cabib, E., Shaw, J. A., Mol, P. C., Bowers, B. \& Choi, W.-J. (1996). In The Mycota, vol. III, Biochemistry and Molecular Biology, pp. 243-267. Edited by R. Brambl \& G. A. Marzluf. Berlin, Heidelberg \& New York: Springer.

Choi, W.-J., Sburlati, A. \& Cabib, E. (1994). Chitin synthase 3 from yeast has zymogenic properties that depend on both the CAL1 and the CAL3 genes. Proc Natl Acad Sci USA 91, 4727-4730.

Chuang, J. S. \& Schekman, R. W. (1996). Differential trafficking and timed localization of two chitin synthase proteins, Chs $2 p$ and Chs3p. J Cell Biol 135, 597-610.

Cos, T., Ford, R., Trilla, A., Duran, A., Cabib, E. \& Roncero, C. (1998). Molecular analysis of Chs3p participation in chitin synthase III activity. Eur J Biochem 256, 419-426.

DeMarini, D. J., Adams, A. E. M., Fares, H., Virgilio, C. D., Valle, G., Chuang, J. S. \& Pringle, J. R. (1997). A septin-based hierarchy of proteins required for localized deposition of chitin in the Saccharomyces cerevisiae cell wall. J Cell Biol 139, 75-93.

Feilotter, H. E., Hannon, G. J., Ruddell, C. J. \& Beach, D. (1994). Construction of an improved host strain for two hybrid screening. Nucleic Acids Res 22, 1502-1503.

Holthuis, J. C. M., Nichols, B. J. \& Pelham, H. R. B. (1998). The syntaxin $T \lg 1 \mathrm{p}$ mediates trafficking of chitin synthase III to polarized growth sites in yeast. Mol Biol Cell 9, 3383-3397.

Ito, H., Fukuda, Y., Murata, K. \& Kimura, A. (1983). Transformation of intact yeast cells treated with alkali cations. J Bacteriol 153, 163-168.

Kawamoto, S., Nomura, M. \& Ohno, T. (1992). Cloning and characterization of SKT5, a Saccharomyces cerevisiae gene that affects protoplast regulation and resistance to killer toxin of Kluyveromyces lactis. J Ferment Bioeng 74, 199-208.

Kollar, R., Petrakova, E., Ashwell, G., Robbins, P. W. \& Cabib, E. (1995). Architecture of the yeast cell wall. The linkage between chitin and beta (1 $\rightarrow 3)$-glucan. J Biol Chem 270, 1170-1178.

Laemmli, U. K. (1970). Cleavage of structural proteins during the assembly of the head of bacteriophage T4. Nature 227, 680-685.

Nagahashi, S., Sudoh, M., Ono, N. \& 7 other authors (1995). Characterization of chitin synthase 2 of Saccharomyces cere- visiae: implication of two highly conserved domains as possible catalytic sites. J Biol Chem 270, 13961-13967.

Sambrook, J., Fritsch, E. F. \& Maniatis, T. (1989). Molecular Cloning: a Laboratory Manual, 2nd edn, pp. 18.60-18.75. Cold Spring Harbor, NY: Cold Spring Harbor Laboratory.

Santos, B. \& Snyder, M. (1997). Targeting of chitin synthase 3 to polarized growth sites in yeast requires Chs5p and Myo2p. J Cell Biol 136, 95-110.

Santos, B., Duran, A. \& Valdivieso, M. H. (1997). CHS5, a gene involved in chitin synthesis and mating in Saccharomyces cerevisiae. Mol Cell Biol 17, 2485-2496.

Sburlati, A. \& Cabib, E. (1986). Chitin synthetase 2, a presumptive participant in septum formation in Saccharomyces cerevisiae. J Biol Chem 261, 15147-15152.

Shaw, J. A., Mol, P. C., Bowers, B., Silverman, S. J., Valdivieso, M. H., Duran, A. \& Cabib, E. (1991). The function of chitin synthases 2 and 3 in the Saccharomyces cerevisiae cell cycle. J Cell Biol 114, 111-123.

Silverman, S. J. (1989). Similar and different domains of chitin synthases 1 and 2 of $S$. cerevisiae: two isozymes with distinct functions. Yeast 5, 459-467.

Silverman, S. J., Sburlati, A., Slater, M. L. \& Cabib, E. (1988). Chitin synthase 2 is essential for septum formation and cell division in Saccharomyces cerevisiae. Proc Natl Acad Sci USA 85, 4735-4739.

Sudoh, M., Tatsuno, K., Ono, N., Chibana, H., Yamada-Okabe, H. \& Arisawa, M. (1999). The Candida albicans CHS4 gene complements a Saccharomyces cerevisiae skt $5 /$ chs 4 mutation and is involved in chitin biosynthesis. Microbiology 145, 1613-1622.

Takita, M. \& Castilho-Valvicius, B. (1993). Absence of cell wall chitin in Saccharomyces cerevisiae leads to resistance to Kluyveromyces lactis killer toxin. Yeast 9, 589-598.

Trilla, J. A., Cos, T., Duran, A. \& Roncero, C. (1997). Characterization of CHS4 (CAL2), a gene of Saccharomyces cerevisiae involved in chitin biosynthesis and allelic to SKT5 and CSD4. Yeast 13, 795-807.

Valdivieso, M. H., Mol, P. C., Shaw, J. A., Cabib, E. \& Duran, A. (1991). CAL1, a gene required for activity of chitin synthase 3 in Saccharomyces cerevisiae. J Cell Biol 114, 101-109.

Yabe, T., Yamada-Okabe, T., Nakajima, T., Sudoh, M., Arisawa, M. \& Yamada-Okabe, H. (1998). Mutational analysis of chitin synthase 2 of Saccharomyces cerevisiae; identification of additional amino acid residues involved in the catalysis. Eur $J$ Biochem 258, 941-947.

Ziman, M., Chuang, J. S. \& Schekman, R. W. (1996). Chs1p and Chs3p, two proteins involved in chitin synthesis, populate a compartment of the Saccharomyces cerevisiae endocytic pathway. Mol Biol Cell 7, 1909-1919.

Ziman, M., Chung, J. S., Tsung, M., Hashimoto, S. \& Schekman, R. (1998). Chs6p-dependent anterograde transport of Chs3p from the chitosome to the plasma membrane in Saccharomyces cerevisiae. Mol Biol Cell 9, 1565-1576.

Received 19 July 1999; revised 27 October 1999; accepted 16 November 1999. 